\title{
Coordination of knowledge in judging animated motion
}

\author{
Thomas C. Thaden-Koch \\ School of Physics and Astronomy, University of Minnesota, Minneapolis, Minnesota 55455, USA \\ Robert J. Dufresne \\ Department of Physics and Scientific Reasoning Research Institute, University of Massachusetts Amherst, Amherst, Massachusetts 01003, \\ USA \\ Jose P. Mestre* \\ Departments of Physics and Educational Psychology and the Beckman Institute, University of Illinois at Urbana-Champaign, Urbana, \\ Illinois 61801, USA
}

(Received 30 December 2005; published 2 November 2006)

\begin{abstract}
Coordination class theory is used to explain college students' judgments about animated depictions of moving objects. diSessa's coordination class theory models a "concept" as a complex knowledge system that can reliably determine a particular type of information in widely varying situations. In the experiment described here, fifty individually interviewed college students judged the realism of two sets of computer animations depicting balls rolling on a pair of tracks. The judgments of students from an introductory physics class were strongly affected by the number of balls depicted (one or two), but the judgments of students from an educational psychology class were not. Coordination analysis of interview transcripts supports the interpretation that physics students' developing physics knowledge led them to consistently miss or ignore some observations that the other students consistently paid attention to. The analysis highlights the context sensitivity and potential fragility of coordination systems, and leads to the conclusion that students' developing knowledge systems might not necessarily result in consistently improving performance.
\end{abstract}

DOI: 10.1103/PhysRevSTPER.2.020107

PACS number(s): 01.40.Fk

\section{INTRODUCTION}

Considerable effort has been devoted to studying students' conceptual understanding of science. There exist large bibliographies on the topic, ${ }^{1}$ as well as detailed studies of specific conceptions possessed by students of different ages. ${ }^{2-6}$ These efforts have been fruitful, but notions of what it means to "know a science concept" remain vague. ${ }^{7}$ There is also a lack of consensus among conceptual change researchers about the nature of students' naive, emergent knowledge. Some support the notion that students possess naive theories that are structured and explanatory, not unlike scientific theories. ${ }^{3,4,8-10}$ Others argue that students' ideas are sufficiently incoherent and context dependent that they are fundamentally nontheorylike. ${ }^{11,12}$ Questions such as "what does it mean to learn and apply a science concept?" and "what is the nature of conceptual change?" are fundamental to science education research, yet disagreements continue about the answers to these questions.

In an effort to make sense of the widely varying "conceptual change" landscape, diSessa and Sherin ${ }^{7}$ introduced the construct of coordination class. Coordination class theory is a step toward a coherent view of what it means to learn and use scientific concepts. Although the coordination class perspective offers a unique lens through which to view the learning and use of physics concepts, to date, published research on its use by the physics education research community has been limited. ${ }^{13-15}$ One of our purposes here is to extend the use of coordination theory to a new sort of situation, one in which the main thrust of students' reasoning is not related to something easily identifiable as a coordination class. We adopt the coordination class perspective to analyze students' conceptual reasoning about the realism of animated motion. We examine interview data of students' judgments concerning the realism of computer animations showing the motion of metal balls rolling on a pair of metal tracks. When presented with an animation of a single ball, most students focused on the presence or absence of realistic speed changes. Addition of a second ball in the animations drastically changed the strategies that students used to determine realistic motion, resulting in (a) an overall shift in what students judged to be realistic motion, and (b) the surprising finding that the majority of students in college-level physics courses appeared to miss a key observation about one of the animations made by nearly all students not taking collegelevel physics courses. A second purpose of this paper is to use a coordination class perspective to explicate these findings. This second purpose is subservient to this paper's potential contribution to the extension and articulation of coordination class analysis. ${ }^{16}$

\section{COORDINATION CLASS CONSTRUCT}

DiSessa and Sherin's original work ${ }^{7}$ provides a detailed introduction to the notion of coordination class, along with a review and critique of other theoretical frameworks for describing scientific concepts and conceptual change. In essence, a coordination class is a type of concept consisting of a complex system of many elements and kinds of knowledge (diSessa and Wagner ${ }^{13}$ comprehensively redescribe the coordination class construct). The primary function of a coordination class is to extract some class of information (that is characteristic of the coordination class) from the world. The coordination class construct has been applied to student's understanding of forces, ${ }^{7}$ waves,${ }^{15}$ and motion..${ }^{14}$ We attempt 
TABLE I. Summary of the main elements of coordination classes. (Adapted from diSessa and Wagner (Ref. 13). Used with permission.)

Level I: Core function

A coordination class is a particular kind of concept whose principal function is to allow people to read a particular class of information out of situations in the world.

\section{Level II: Intrinsic difficulties}

We assume that reading the same information out of a wide range of situations poses core problems for developing a coordination class.

Span: A learner must accumulate enough knowledge to "operate" the concept across the full range of contexts in which it is applicable.

Alignment: The information determined in different situations, possibly using different knowledge, must be the same information.

Level III: Architecture

Readout strategies: Readout strategies are the ways in which people focus their attention and read out information relevant to, but possibly not the same as, the defining information.

Causal net: The causal net is the set of inferences that people use to infer defining information from related kinds of information. We can distinguish two classes of inferences:

Inferences related to the mere existence of the coordination class, and its relevance to what is required or problematic in a situation.

Inferences related to specific determination of the coordination class's defining information.

a thorough application of coordination classes to a case involving students' judgments about the realism of animated motion. Unlike previous applications of the coordination class construct in physics to explicate students' understanding of specific concepts, our application of coordination class to study students' perception of realism is unique in its level of abstraction. That is, although students in this study will use physics concepts that might function as coordination classes for experts (e.g., velocity and velocity changes, conservation of energy), the focus of this investigation is to explore the usefulness of coordination classes in understanding students' judgments of realism of motion. We begin with a brief overview of the coordination class construct, which is summarized in Table I.

\section{A. Performance specifications of a coordination class}

We have stated that the purpose of a coordination class is to extract information of some characteristic class from the world. A coordination class must meet two performance criteria. The first is to determine the characteristic class of information across the wide variety of situations in which such a determination is possible. The term span describes the full range of contexts over which the coordination class can be applied. The second performance criterion for a coordination class, alignment, is the ability to reliably determine the same information across its span. If the information determined across different contexts is not the same type of information, there is a problem with alignment. Note that these are requirements about the coordination class's input range (situations in the world) and output type (characteristic information); they say nothing about the structure or operation of a coordination class.

\section{B. Methods and structure: Knowledge components of a coordination class}

A coordination class contains knowledge sufficient for making observations in a situation, and for making inferences based on the results of those observations. Readout strategies refer to the part of the complex knowledge system responsible for making observations of the world. The information obtained directly by a readout strategy may, or may not, be the information characteristic of the coordination class, but is somehow useful to the coordination class for determining its characteristic information. A coordination class may call upon multiple readout strategies in a single situation, and it may call upon different sets of readout strategies in different situations. We refer to the information produced by a readout strategy as a readout.

The part of the complex knowledge system responsible for making inferences that support the functioning of a coordination class is the causal net. The causal net is the knowledge used to (a) determine which readout strategies could be useful in a situation, and (b) make inferences based on readouts to determine the output of the coordination class (i.e., the information characteristic of the coordination class) in a particular situation.

It should be pointed out that a coordination class (or coordination system - see next section) may use other coordination classes (or coordination systems) as intermediate steps for inferring the class's characteristic information. For example, in "running" the coordination class for acceleration, a person might call on the coordination classes of speed and time duration, and deduce acceleration information from their outputs. We will see examples of this in the data presented later.

Achieving alignment across a suitable span requires that the readout strategies used to make readouts, and the causal 
net elements used to draw inferences from those readouts, be appropriate to each situation in the span. In general, it is expected that achieving alignment across a wide span will require a wide range of knowledge. Some of that knowledge may be more closely tied to particular types of situations (knowledge about reading a speedometer to determine speed, for example) than to an abstract understanding of the information characterizing a particular coordination class (a verbal definition of speed, for example).

\section{Coordination class vs coordination system}

Coordination classes, as defined by diSessa and his collaborators, must meet strict performance specifications. Experts in a domain may have concepts that meet these specifications. It is unlikely, however, that novices possess knowledge systems that meet the requirements of fully functioning coordination classes. Nevertheless, novices' knowledge systems may be entirely adequate for many situations. Undoubtedly, they can coordinate observations and other knowledge to produce some class of information, but with limited span and/or alignment problems. Following Thaden-Koch ${ }^{17}$ we will use the term coordination system to describe such knowledge systems.

\section{Projections}

When a person needs to determine a piece of knowledge (of a type characteristic of some coordination class) in a situation, the person brings to bear some particular set of strategies and cognitive operations. diSessa and Wagner ${ }^{13}$ refer to such a set as a concept projection. Thus, a concept projection is the subset of causal net elements and readout strategies from the coordination class that is evoked by a particular situation. Note that, for a person whose relevant coordination system is not a well-formed coordination class, successful use of a concept in one context does not mean successful application in other contexts.

\section{EXPERIMENT}

\section{A. Task}

Two groups of students were asked to judge the realism of the simulated motion of steel balls moving along metal tracks. The apparatus is shown in Fig. 1.

The first group consisted of 26 students enrolled in an educational psychology course at a large state university. The second group consisted of 24 students enrolled in an honors calculus-based introductory physics course at the same university. We will refer to these as the "psychology students" and the "physics students," respectively. Each student was interviewed individually and given control of a computer where they could play five animations; students could view the animations as many times as they wished, in regular speed or slow motion. During the interviews students were asked to "think aloud" as they reasoned about the realism of the animations; the interviews were tape-recorded.

The students judged two different sets of animations, with each set containing five animations. The first set of five (oneball) animations showed a single ball moving along track B;

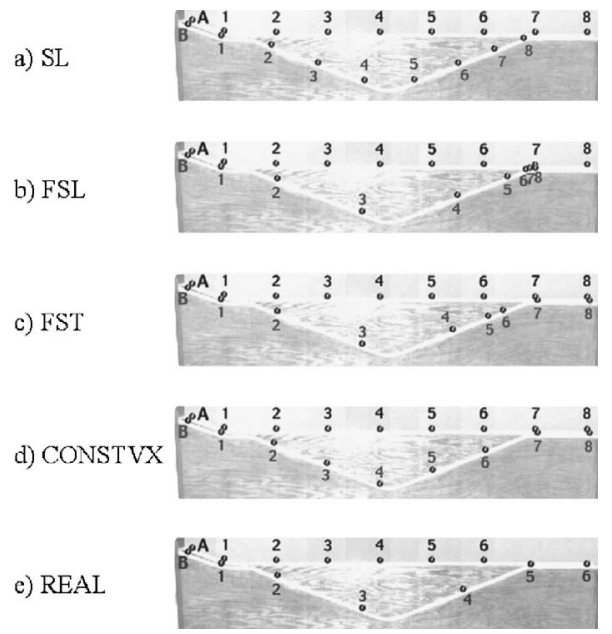

FIG. 1. Strobe diagrams indicating positions of balls on tracks A and B in every ninth frame of the two-ball animations (QuickTime movies). The motion of ball $\mathrm{B}$ varies across animations after it enters the valley. (a) Slow-lose (SL): ball B lags behind ball A. (b) Fast-slow-lose (FSL): ball B experiences an exaggerated deceleration on the final slope. (c) Fast-slow-tie (FST): while rolling uphill, ball B slows down and then speeds up. (d) Constant-Vx (CONSTVX): the balls are always tied. (e) Realistic (REAL): realistic motion for ball $\mathrm{B}$.

the second set of five (two-ball) animations showed both balls moving in a race. Figure 1 shows a strobe representation of the two-ball animations. In each two-ball animation, the motion depicted for the ball on track A was realistic. The one-ball animations, excepting the absence of the ball on track A, were the same as the two-ball animations. Figure 1 includes descriptions of the motion, as well as labels that describe the motion of ball B relative to ball A; for example, in "fast-slow-lose" (FSL) ball B speeds up realistically on the downhill slope of the $\mathrm{V}$, but slows down excessively on the uphill slope of the $\mathrm{V}$, so that ball $\mathrm{B}$ loses the race. In fast-slow-tie (FST), ball B speeds up realistically on the downhill portion of the $\mathrm{V}$, and slows down on the uphill portion with a brief but noticeable period where the ball speeds up on the uphill portion (about $2 / 3$ of the way up the slope); this animation results in a tie. The realistic animation is labeled REAL in Fig. 1.

\section{B. Aggregate animation preferences}

The number of students selecting each of the animations as realistic is displayed by context (one ball vs two ball) for physics students in Table II and for psychology students in Table III. Note that entries on the diagonal for each table correspond to students who chose the same motion consistently across the one-ball and two-ball contexts. Our analysis will focus on two outcomes: (1) Changing the context from one ball to two balls resulted in a pronounced difference in the selection pattern for the physics students; and (2) the selection patterns for the two groups were broadly similar in the one-ball context but there were marked inter group differences in the two-ball context. 
TABLE II. Animation preferences for one- and two-ball tasks; 24 students from introductory calculusbased physics (honors) course.

\begin{tabular}{|c|c|c|c|c|c|c|}
\hline \multicolumn{7}{|c|}{ Number choosing one-ball and two-ball animations as realistic } \\
\hline & $\begin{array}{l}\text { Two-ball } \\
\text { SL }\end{array}$ & $\begin{array}{l}\text { Two-ball } \\
\text { FSL }\end{array}$ & $\begin{array}{c}\text { Two-ball } \\
\text { FST }\end{array}$ & $\begin{array}{l}\text { Two-ball } \\
\text { CONSTVX }\end{array}$ & $\begin{array}{l}\text { Two-ball } \\
\text { REAL }\end{array}$ & $\begin{array}{c}\text { (One-ball) row } \\
\text { total }(\%)\end{array}$ \\
\hline One-ball SL & & & 1 & 1 & & $2(8 \%)$ \\
\hline One-ball FSL & & 3 & 7 & & & $10(42 \%)$ \\
\hline One-ball FST & & & & & & $0(0 \%)$ \\
\hline One-ball CONSTVX & & & & & & $0(0 \%)$ \\
\hline One-ball REAL & & 1 & 7 & 3 & 1 & $12(50 \%)$ \\
\hline (Two-ball) column total $(\%)$ & $0(0 \%)$ & $4(17 \%)$ & $15(63 \%)$ & $4(17 \%)$ & $1(4 \%)$ & $24(100 \%)$ \\
\hline
\end{tabular}

We describe the one-ball selection patterns first. In the one-ball context the majority of students in both groups selected either the REAL animation or FSL as realistic. Recall that the FSL animation has features that resemble realistic motion, such as ball B speeding up on the downhill portion of the $\mathrm{V}$ and slowing down on the uphill portion; however, this animation is unrealistic in that the ball's acceleration on the uphill slope is greater in magnitude than its acceleration on the downhill slope. Fewer students from either group selected one-ball animations with other unrealistic features; in particular not a single student from either group picked the FST animation as realistic (it depicts ball B speeding up going uphill). Only a single psychology student selected the one-ball CONSTVX animation as realistic (ball B moves with constant horizontal speed). Animation SL (in which ball B slows down going uphill but does not speed up going downhill) was more popular among psychology students than among physics students.

The selection pattern for the psychology group was similar for the two contexts (one ball and two ball), but this was not the case for the physics group. For the physics students, by far the most popular two-ball choice was FST, where the balls tie but where ball B undergoes anomalous motion in the uphill section of the V; apparently most of the physics students did not see the ball speeding up going uphill, or they saw it and disregarded it. The other two most popular selections for the physics students were the second animation where the balls tied (CONSTVX), and an animation with several realistic features (FSL). Note that the REAL animation was selected by fewer than $5 \%$ of the physics students in the two-ball task (compared to $50 \%$ in the one-ball task).

\section{Students' approach to the tasks}

Based on the interviews, we have developed the following description of students' general approach. From observations of the shape of the tracks, and perhaps observations of the motions presented in the animations, students formed expectations of realistic motion for balls on the tracks. In the oneball animations, for example, the majority of students explicitly indicated that they expected ball B to speed up while going downhill along the $\mathrm{V}$, and to slow down while going uphill on the V. Students also had expectations about the race outcome in the two-ball animations; for example, the majority of physics students expected the balls to tie. Students observed the motion of the balls, and checked those observations against their expectations. Students made both accurate and inaccurate observations. Students also made decisions about the realism of the animations that appeared to be based on a set of expectations and observations that, despite individual differences, were remarkably similar across students. The majority of expectations appeared to be correct (compatible with realistic motion). (Table IV lists the most common expectations.) When considering a particular animation, students typically described the comparison of a small number of expectations (very often one or two) against

TABLE III. Animation preferences for one- and two-ball tasks; 26 students from an educational psychology class.

\begin{tabular}{|c|c|c|c|c|c|c|}
\hline \multicolumn{7}{|c|}{ Number choosing one-ball and two-ball animations as realistic } \\
\hline & $\begin{array}{c}\text { Two-ball } \\
\text { SL }\end{array}$ & $\begin{array}{c}\text { Two-ball } \\
\text { FSL }\end{array}$ & $\begin{array}{c}\text { Two-ball } \\
\text { FST }\end{array}$ & $\begin{array}{c}\text { Two-ball } \\
\text { CONSTVX }\end{array}$ & $\begin{array}{c}\text { Two-ball } \\
\text { REAL }\end{array}$ & $\begin{array}{c}\text { (One-ball) row } \\
\text { total }(\%)\end{array}$ \\
\hline One-ball SL & & 3 & & 1 & 2 & $7(23 \%)$ \\
\hline One-ball FSL & 2 & 3 & & & 1 & $9(35 \%)$ \\
\hline One-ball FST & & & & & & $0(0 \%)$ \\
\hline One-ball CONSTVX & & & & 1 & & $1(4 \%)$ \\
\hline One-ball REAL & 2 & 2 & & & 6 & $10(38 \%)$ \\
\hline (Two-ball) column total $(\%)$ & $4(15 \%)$ & $11(42 \%)$ & $0(0 \%)$ & $2(8 \%)$ & $9(35 \%)$ & $26(100 \%)$ \\
\hline
\end{tabular}


TABLE IV. Expectations commonly expressed about realistic motion in the one-ball and two-ball tasks.

Expectations about speed changes

DECELUP Speed should decrease rolling uphill.

ACCELDOWN Speed should increase rolling downhill.

SAMESPEED Ball B should have the same speed before and after the valley.

NOGAIN

Ball B should not gain speed without an apparent cause.

Expectations about race outcome

TIE Balls reach the end simultaneously.

B WIN Ball B wins.

B LOSE Ball B loses.

the observed motion of the ball(s) on some portion of the track. An animation might be accepted (rejected) on the basis of a single observation being compatible (incompatible) with a single expectation. Inaccurate observations often led students to reject features of animations that were realistic (and that might well have matched their stated expectations) and to accept features of animations that were unrealistic (and might have violated their stated expectations). Occasionally, students' expectations and observations led them to claim that none of the animations depicted realistic motion; students in that predicament had to reevaluate their expectations or their observations or both.

\section{Relationship of interview data to coordination class construct}

As pointed out in the Introduction, our primary purpose concerns extending and articulating coordination theory. In the following sections, we attempt to do that by exploring whether and how coordination theory can be used to (at least partially) explicate the results described above.

\section{Identifying coordination system outputs}

By requesting that students answer the question "Which animation depicts realistic motion?" we instructed students to achieve a final output for their efforts at (what we describe as) coordination - the output of their coordination is a reference to the animation that, to them, depicts realistic motion. Within a limited set of contexts, they had to remember knowledge and develop observation strategies for achieving the requested output. In principle, one might try to imagine a coordination class capable of recognizing realistic motion in every circumstance. In practice, however, it is easier to imagine an individual bringing a set of knowledge and strategies to bear on identifying realistic motion in the task provided-a coordination system that extracts information about the realism of motion depicted in animations of balls rolling on these particular ramps. The input for such a coordination system is some set of animations; the output is a "yes" or "no" for each animation, with the restriction that there be precisely one "yes" per set of animations. From students' verbal reports, we attempted to identify causal net elements and readout strategies brought to bear on analysis of a particular animation (or set of animations).

The central strategy of nearly all the students was to break the over-arching question about animation realism into smaller questions about characteristics of the animations, questions more localized than the general "realism" question. Students determined characteristics of realistic motion useful for judging a given animation. The motion in the animation was tested for realism by observationally testing for that set of characteristics. Typically, this involved coordinating information about speed, speed changes, relative motion and position. From this viewpoint, we can describe a top-level coordination system (trying to answer the "realism" question) making use of other coordination systems to extract some types of information from the situation.

We begin by identifying potential coordination systems based on student outputs. We will describe three; we do not mean this list of three to be exhaustive, but intend it as an illustration of three different levels of coordination system candidates. A candidate for the highest-level coordination system is realism of motion. In running the coordination system for realism of motion an individual would make observations of the shape of the track, form expectations about the motion of the ball(s), determine whether the speed and speed changes of the ball(s) meet the expectations, and select an appropriate animation as realistic. A second candidate, needed to characterize the motion in order to address subquestions posed by the highest-level coordination system and hence lower in the hierarchy, is speed and speed changes. This would entail observing a ball and determining if it is moving at a constant speed, speeding up, or slowing down, and in some cases evaluating the magnitudes of the speed changes (in comparisons with expectations are they too small or exaggerated, or are they realistic?). A third candidate, even lower in the hierarchy, would be position, for which information is obtained by direct observation of the ball. As stated earlier, a coordination system, such as realistic motion, can employ other coordination systems (such as speed and speed changes and position) to make inferences. Examples are now provided to illustrate manifestations of some of these coordination system candidates from student interviews.

Example 1. Student 1 (physics student, female) stating why she rejects FST and accepts FSL in the one-ball animations.

S1: Number ${ }^{18} 4[\mathrm{FST}]$ it looks like it, stops threequarters of the way up the $\mathrm{V}$ and then accelerates again before it goes over the bump, so that's wrong.

$\langle$ Later in the same task

S1: I liked number 2[FSL] because it started off too, it started off getting a little bit faster as it went down the first ramp, and then as it went down the $\mathrm{V}$ it got a lot faster and as it went up the $\mathrm{V}$ it slowed down a little bit, and then when it got to the level part at the end it pretty much had the same speed as the little part of level part at the beginning.

Interviewer: And that's good?

S1: Uh huh.

Interviewer: Why do you like that, why do you think it's...? 
S1: Um, because they're at the same height, so they should have the same speed....

Example 1 indicates that from the shape of the track Student 1 forms expectations of realistic motion of the ball, including constraints on the speed changes permissible in particular sections of the track, and relationships among speeds on two separate segments of the track. When readouts violate expectations, she rejects animations (e.g., she notes that FST violates the NOGAIN expectation), and when readouts match expectations, she selects an animation as realistic (e.g., she determines that FSL meets her ACCELDOWN, DECELUP, and SAMESPEED expectations).

Example 2. Student 2 (physics student, male) stating why he accepts FSL in the one-ball animations.

S2: Well, number 2[FSL], well, what I was looking for in each one, there has to be, some sort of acceleration that's heading downhill, and it's got to decelerate when it's going uphill, um, similar to the same, like, energy argument that I uh used before, and at points where it's the same, uh at the same point, the velocity would have to be the same.

Example 2 illustrates the types of subquestions generated by the student, and concept projections referenced, leading to the selection of the FSL one-ball animation as realistic. Student 2 describes the ACCELDOWN, DECELUP, and SAMESPEED expectations for the ramp, and implies that they have led him to deploy readout strategies for extracting information about (reading out) particular speeds and speed changes depicted in the animations. Without explicating readout strategies or causal net elements of the concept projection(s) used to make the readouts, he states that FSL meets each of those expectations. He claims to have inferred the SAMESPEED expectation with readouts about the track shape and his (causal net) knowledge about conservation of energy - when the ball is at the same point (from a previous explanation it was clear that by "point" he meant "height") it will have the same speed.

In both of these excerpts, inaccurate readouts have evidently led to the students accepting animations with features that violate their stated expectations-in the FSL case, the ball's motion fails to satisfy SAMESPEED. This also was common in the two-ball judgments, but in addition, the TIE expectation took on primary importance for the physics students, as the following excerpt from Student 3 demonstrates.

Example 3. Student 3 (physics student, male) demonstrating reasoning leading to selection of FST as realistic in two-ball animations.

S3: Yeah, I think they should end up at the same, the same spot. 'Cause I think from the last one 3[FST] and $4[$ CONSTVX] were pretty close, so this one I'm going to be looking for if they show up at the same spot, the first one [SL] they didn't, the second one [FSL] they didn't, again, 〈longer pause $\rangle$ the third one [FST] it did $\langle$ similar pause $\rangle$ fourth one [CONSTVX] did, fifth one [REAL] didn't. Again, I'm still not, I'm pretty sure they should end up at the same spot, but I don't want to discount, I don't want to, like automatically say since they didn't show up at the same spot that it's absolutely wrong, so, so $3[\mathrm{FST}]$ and $4[$ CONSTVX] again are the ones that end, they ended up at the same spot. So go back to $1[\mathrm{SL}]$. 〈clicks〉 In $1[\mathrm{SL}]$ it really doesn't look like it speeds up at all going down the first ramp so I don't think $1[\mathrm{SL}]$ 's right, the first ball, the one that's closer to me. 〈clicks〉 The second one [FSL] again, it looks pretty accurate but they don't end up at the same spot, so I'm again I'm inclined to think that that one's not right. 〈clicks〉 Third one [FST] looks like the second one [FSL] except they end up at the same spot. The second one [FSL] looked very accurate but they didn't end up at the same position together. But the third one [FST], it also looked accurate and they ended up at the same spot, so I'm right now I think third one [FST] is the best one. Fourth one [CONSTVX] they ended up at the same spot, but it really doesn't look like the ball that's closer to me has any sort of acceleration at all, it should speed up going down the hill and slow down going up. Actually, the more I look at it they stay at the same position the whole time. So I don't think the fourth one's right. The fifth one [REAL] it really doesn't look like it slows down coming up the incline, so I don't think that one's right, plus there's a huge gap between the two balls. So I would think the third one [FST] is right.

Example 3 provides evidence of a student's attempt to use the three candidate coordination systems mentioned earlier: Is each animation depicting realistic motion? What are characteristics of speeds and speed changes depicted? What are characteristics of positions depicted? Here, however, the TIE expectation (related to position) dominates, and he continually looks for the balls to end up at the same position at the end of the race. He dismisses several animations by stating that they violate expectations about speed changes, and eventually ends up selecting FST stating, "it also looked accurate and they ended up at the same spot." Note that this student selected REAL as the most realistic one-ball animation and said that the one-ball FST animation violated NOGAIN (see the following excerpt from the same student)

Example 4. Student 3 (physics student, male) dismissing FST and selecting REAL in one-ball animations. S3: That one [FST] looks like it almost stops, and then speeds up for some strange reason.

Interviewer: Where?

S3: Right in the middle of the ramp going up. 〈clicking See, it almost stops, like two thirds of the way up. 〈clicks〉 That doesn't make any sense either, where it would just speed up for no reason. 〈clicks〉3[REAL] looks pretty good. Definitely goes faster, it's, it's going fastest at the bottom of the hill, the bottom of the V.

\section{Link to readout strategies and causal net}

Coordination classes have two main componentsreadout strategies and the causal net. We discuss three candidates for readout strategies within the two tasks. The first is observe shape of track. That is, students look at different sections of the tracks (for example, to qualitatively determine 
characteristics of realistic ball speed on those sections, which will link information about speed and speed changes to judging realism of motion). Note that parts of students' coordination systems can be quite adequate-in this case students have no difficulty reading out the shape of the track by direct observation to the extent such readouts were necessary for judging realistic motion. Another candidate is observe qualitatively the speed and speed changes against a fixed background. This strategy, which we will refer to as fixed referent, was common in the one-ball animations where students had only the track's fixed background against which to make readouts regarding speeds and speed changes. (Examples 1 and 2 indicate fixed-referent readout strategies.) In the twoball cases students could also observe position and speed changes of the balls relative to each other. Students used this readout strategy, which we will refer to as relative motion, to infer readouts about speeds from readouts about the positions of the balls relative to each other. Student 3's description of two-ball animations in Example 3 demonstrates the use of both fixed-referent and relative motion readout strategies (the latter is most clearly evident in his rejection of REAL at the end of the quote, stating "there's a huge gap between the two balls").

Recall that the causal net is used to determine which readout strategies could be useful in a situation and to make inferences based on readouts to determine the output of the coordination class. Potential candidates for components of the causal net are expectations about the speed changes of a ball on different regions of the track, and expectations about the relative position of the two balls in the context of the race. Note that the causal net can allow more complex inference-making than simply using a single expectation to reach a conclusion about the realism of motion depicted in an animation. Examples 1-4 show that students use both single expectations and multiple expectations to accept or reject individual animations.

\section{Link to span and alignment}

Span and alignment are performance specifications for coordination classes. If a coordination class can determine a characteristic class of information reliably across some suitable range of situations then it has appropriate span. Alignment refers to determining the same information across the span. We present interview data from two physics students to illustrate problems with both span and alignment. The first case involves Student 3 (Examples 3-6). In the one-ball cases, his projection of a coordination system for realism of motion relies on determining speed and speed changes. He dismisses animations observed to violate expectations and accepts the one-ball REAL animation as most realistic (Examples 4 and 5).

Example 5. Student 3 (physics student, male) demonstrating procedure for analyzing realistic motion in the one-ball animations.

S3: OK. First one [CONSTVX] 〈pauses〉 looks like it has the same speed going down along the $\mathrm{V}$ ramp as it does going up. It shouldn't. It's constant speed. On the first one. That one [FST] looks like it almost stops, and then speeds up for some strange reason.
Interviewer: Where?

S3: Right in the middle of the ramp going up. 〈clicking $\rangle$ See, it almost stops, like two-thirds of the way up. 〈clicks〉 That doesn't make any sense either, where it would just speed up for no reason. 〈clicks〉3[REAL] looks pretty good. Definitely goes faster, it's, it's going fastest at the bottom of the hill, the bottom of the V. 〈clicks〉 Number 4[SL], again constant speed, it's the same as number 1 but slower. 〈clicks〉 Looks constant. Interviewer: Looks what?

S3: Looks, yeah, maybe it does slow down. It doesn't really seem like it speeds up much going down hill, it just doesn't look right, goes faster, it still looks like, it should be going really down at the bottom and then much slower then at the top, and this one there's not much change in speed. 〈clicks〉 That was 4[SL]. 〈clicks〉Ah, number 5[FSL], it pauses at the top, it looks like it stops at the top, but then keeps rolling. The top of the crest, like it's almost about to roll back. It doesn't seem like it should just, start rolling again after it reaches the top, because it, it goes down, hits the $\mathrm{V}$, goes up, and then ought, looks like it pauses for a moment, and then starts rolling again, but it's a flat surface so it shouldn't, almost pause and then start up again.

In contrast, Student 3's approach to determining realistic motion in the two-ball animations (Example 3) is dominated by the TIE expectation. Although he attempts to evaluate speed and speed changes, he makes different readouts than he did in the one-ball cases. In particular, he rejects REAL in the two-ball case as violating the TIE and ACCELUP expectations, whereas he judged REAL to be the most realistic in the one-ball animations in terms of speed and speed changes; he accepts FST in the two-ball animations since it "looked accurate" and satisfied TIE, whereas he noticed that FST violates NOGAIN in the one-ball case and dismisses it as unrealistic. In each situation he came to a judgment and was satisfied with that judgment. Focusing almost exclusively on a single expectation at the expense of other useful expectations, failing to activate relevant expectations in the two-ball case that were activated in the one-ball case, and using different readout strategies in the one-ball and two-ball cases, all contribute to lack of span and alignment.

An important point to stress is that this student's performance was not simply inconsistent in a general way. Rather, it was inconsistent in a way that can be explained in terms of the coordination system we posit for him. When asked by the interviewer to justify the TIE outcome in the two-ball task, he provides the following explanation based on conservation of energy.

Example 6. Student 3's justification of the TIE expectation.

S3: Well, well, it's from physics, the, the kinetic energy and the potential energy, it has to, has to have the same energy at the end, so at the top, they both have the same kinetic energy, then they go down, and they both have same energy up until the point where they deviate, and then one gains more potential energy and then 
loses that potential energy so it should actually have the same energy at the end 'cause they're both at the same height. If they were at different heights, they'd be, one would be farther ahead than the other but since they go back up to the same height and, like the friction is the same on both tracks they really should be at the same spot 'cause they should both still have the same energy. ${ }^{19}$

This student (despite apparent misstatements about whether potential energy was gained or lost) reaches a correct conclusion about the ball's kinetic energy on either side of the valley, and about the ball having equivalent speeds at equivalent heights (the SAMESPEED expectation). However, Student 3 incorrectly infers that having the "same energy" means that the two balls arrive at the end of the track at the same time. The projection of the student's causal net evoked by the two-ball situation supports the incorrect TIE expectation.

It should be noted that expectations and readout strategies related to the balls' relative positions during the race are neither inherently incorrect, nor inferior to expectations and readout strategies related to speeds and speed changes. In the cases studied, however, students were more likely to generate incorrect expectations about realistic positions (notably, TIE) than about realistic speeds and speed changes. Conversely, they were less likely to generate inaccurate readouts of positions than of speed changes. It should also be noted that the causal net directs attention to what might, or should be, observed. In this case, the TIE expectation directs attention to readout strategies that focus on detecting a tie outcome. Coordination class theory does not explain how students' causal nets and readout strategies develop, or are used to arrive at particular inferences (e.g., that conservation of energy implies a tie outcome).

A similar lack of span and alignment is demonstrated by Student 2 in the excerpts below.

Example 7. Student \#2 (physics student, male) demonstrating different approaches for judging realistic motion in the one-ball and two-ball animations for the FST case.

〈Discussing the one-ball FST case〉

S2: It accelerates at, once it gets, it goes down this $\mathrm{V}$ and then goes back up, it seems to accelerate right here.

Interviewer: So just to record, you're pointing about two-thirds of the way up this second part of the V.

S2: Yeah. It seems to slow down and then for some reason it picks up some speed.

〈Discussing the two-ball FST case

S2: The balls start at the same height and then are released; at the point where they split, the one that goes down the $\mathrm{V}$ accelerates so that it um is a little bit ahead of the ball that is on the flat track linearly, but then when it goes up the uh, the uphill part of the $\mathrm{V}$ it decelerates again to the point where um, it meets with the the uh ball that was, that was just on the flat track because they have the same um amount of energy and should uh be together because they were at the same height; it doesn't matter that it went down and then went back up, the uh acceleration and deceleration should cancel each other out.

\section{E. Interpreting selection patterns in view of coordination classes}

There remain two results that merit discussion. (1) The selection differences between the one-ball and two-ball animations for the physics students, and (2) the selection differences between the physics and psychology students in the two-ball animations. These differences are discussed in this section, with evidence for the type of reasoning used by the psychology students that led to these differences.

\section{Selection differences: One-ball vs two-ball animations for the physics students}

Interview data suggest that two strongly interacting factors contribute to the difference between one-ball and twoball selections for physics students. The first relates to differences in the causal net elements evoked in concept projections for the one-ball and two-ball contexts. In the oneball context, physics students developed expectations about realistic speeds and speed changes on sloped and flat portions of the ramps. In the two-ball task, however, physics students placed primary importance on expectations about the balls' relative positions at different positions on the tracks, and especially on the TIE expectation about race outcome, which they quite often justified with conservation of energy-related causal net elements.

As stated earlier, one of the two primary functions of the causal net is to determine which readout strategies could be useful for determining the information required in a situation. In other words, the causal net elements evoked in a situation determine which readout strategies will be used. A prime example of this function is that the TIE expectation leads so many physics students to employ a readout strategy useful for detecting a tie outcome. Thus, differences in readout strategies (the second factor in the differences between one-ball and two-ball choices made by physics students) are strongly related to the first factor. Interview transcripts suggest that students relied exclusively on fixed-referent readout strategies for judging speed changes in the one-ball animations, but that they used a combination of relative-motion and fixed-referent readout strategies for judging speed changes and ball positions in the two-ball animations.

We do not claim that physics students relied solely on the TIE expectation in the two-ball task; two different animations resulted in a tie, and physics students selected FST much more frequently than CONSTVX. We do claim that expectations and readouts about the relative positions of the balls influenced physics students' judgments about speed changes. Three examples support this view. When students compared the two tying animations, CONSTVX and FST, they very often commented that the CONSTVX animation was unrealistic because the balls were always beside each other (see Example 3). A second example is that physics students often judged ball B in the two-ball REAL animation as violating DECELUP on the uphill portion of the $\mathrm{V}$, and related that violation to the balls' relative positions, even 
when they had accurately judged that the one-ball REAL animation met the DECELUP expectation (contrast student 3's statements about two-ball REAL in Example 3 with his one-ball REAL statements in Example 4). Finally, we believe that the strong TIE expectation and the dependence on relative-motion readouts even for judging speed changes led a large fraction of physics students to miss seeing ball B speeding up while going uphill in the FST animation (again, contrast student 3's one-ball and two-ball discussions). Some $92 \%$ of the physics students explicitly reported the NOGAIN violation in the one-ball FST animation, whereas only $25 \%$ reported having seen it in the two-ball case; this is a dramatic demonstration that what students look at (and actually "see") is related to what they take to be most relevant and available (in this case, position is both relevant to TIE and available in the two-ball animations). In fact, even in cases where the physics students reported having observed the NOGAIN violation in the two-ball FST animation, often they would override it in favor of the keeping the TIE expectation (see Dufresne, et al. ${ }^{19}$ for a discussion of this phenomenon). This exemplifies the modification of expectations or readouts by students who had judged all five animations in a set as nonrealistic. The excerpt below from a physics student illustrates the phenomenon, and the conflict that results when students with insufficient span and alignment draw conclusions using different projections that they explicitly recognize as being contradictory.

Example 8. A physics student during the two-ball task, after rejecting all animations except FST.

Physics student: So that leaves me with number 2[FST]. I think, well, except it accelerates a little bit at the end doesn't it, damn it. Why does it do that? Maybe I don't like any of them. I don't think I like any of them 〈laughs〉. 2[FST]'s the closest. ...I think that number $2[\mathrm{FST}]$ is the closest. I just think there's something wrong with having it accelerate at the end like that you know?

\section{Selection differences: Physics vs psychology students in the two-ball task}

For psychology students, the causal nets evoked by the two-ball context (in contrast to those of the physics students) did not place primary importance on the TIE expectation. When psychology students described the TIE expectation, they would present it somewhat weakly, as a free-standing idea, and not as something strongly integrated with other parts of their causal net related to realistic motion (in particular, without using conservation of energy to justify the tie outcome). The excerpt below illustrates a psychology student reaching a predicament similar to that exemplified by the physics student just quoted. This student also resolves the issue by re-evaluating the reliability of causal net elements, but comes to a different conclusion.

Example 9. A psychology student during the two-ball task, after rejecting all animations except FST.

Psychology student: Yeah, which would I think bring

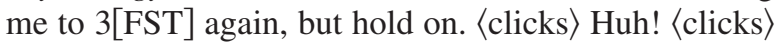
Now that one, that one's the one that actually speeds up, going up the hill. 〈clicks〉 Oh, hmm! What I'm thinking with this one, is, um, I think I liked this, this speed the last time, although I was kind of hesitant that it took so slowly to get over there....

But, I don't like that fact that these [FSL] don't meet, like I think they are supposed to meet at the end, which they do, in this one, the one before it [FST], but to me it still visually looks like it's going more quickly—-too quickly, at the end....

I'll look at again, but, I guess I think that $4[\mathrm{FSL}]$ is the more realis-〈pauses is the most realistic. 〈clicks〉 Hmm definitely not that one [REAL]. 〈clicks〉 It doesn't slow down enough going on top. 〈clicks〉 Yeah, I am going to have to stay with 4 [FSL].

Examples 8 and 9 illustrate how physics and psychology students whose readouts and expectations were at least superficially similar could make different judgments about the animations. A more common difference, however, appeared to lie in the specific readouts made by psychology students and physics students. Both physics and psychology students gave signs of combining relative-motion and fixed-referent readout strategies in the two-ball animations, but psychology students were far more likely than physics students to observe and comment on the NOGAIN violation in the two-ball FST animation. In fact, the frequency of psychology students stating during the interview that they had noticed ball B speeding up going uphill was similar between the one-ball and two-ball cases $(79 \%$ versus $71 \%)$. [Recall that this was not the case for physics students (92\% versus 25\%).] This particular causal net difference between the physics and psychology students (more weight given to a TIE expectation) apparently led to physics students' using a combination of readout strategies that was relatively insensitive to the anomalous motion of ball B in the two-ball FST animation, and to striking differences in the way students from the two groups coordinated information about the same visual stimuli. (Compare, for example, the psychology student quoted above with physics student 3 in Example 3.)

In summary, psychology students used a different combination of readout strategies and gave different weight to information they extracted from the situation, based on the different weights they gave to their expectations. This led psychology students to universally reject the two-ball FST animation, and to select FSL and REAL with a much higher frequency than physics students.

\section{F. Overview of coordination class analysis for the two-track motion judgment experiments}

The complex knowledge systems approach inherent in coordination classes is useful for interpreting our interview data, and in explaining the students' judgments about realistic motion. Students employed various readout strategies (fixed referent, relative motion) and causal net elements [expectations about speed, speed changes, and relative ball position; application of both formal physics knowledge (conservation of energy) and informal knowledge about realistic motion]. Their use and the emphasis placed on them were highly dependent on context (one ball vs two ball). Students' expectations about realistic motion on the tracks were, for 
the most part, compatible with realistic motion, especially those expectations related to speed and speed changes on the various sections of the tracks. There were often inaccuracies in readouts which, when coordinated with correct expectations, led to inaccurate judgments of realistic motion. For example, the DECELUP expectation was almost universally explicated by students, but readouts about speed changes on the uphill portion of the track were often inaccurate (e.g., students judging ball $\mathrm{B}$ not to be slowing enough - or at all-in the REAL animation). The most common exception to this rule was the unrealistic TIE expectation held by most of the physics students, and a large fraction of psychology students, in the two-ball race (note that readouts about race outcome were always accurate). In addition, interview data of the physics students strongly suggests that causal net elements can impact readouts-a strong TIE expectation apparently led the majority of physics students to use readout strategies insensitive to ball B speeding up while going uphill in the two-ball FST animation.

Despite the difficulties many students faced in accurately determining which animation depicted the most realistic motion, most of their individual acts of coordinating information about the characteristics of realistic motion for balls on these tracks, about determining speed and speed changes, and about determining position produced correct information. Reaching a final judgment, consistent across the oneball and two-ball contexts, that a single animation depicted motion consistent with all of their stated expectations, required a level of alignment beyond the capabilities of nearly all the students' coordination systems. So many causal net elements and readouts need to be coordinated accurately to result in a correct judgment of the realism of a given animation that it only takes one thing (or a very small number of things) not working properly for the entire concept projection to fail. Context sensitivity of concept projections exacerbates accurate coordination, since it was common to see very different concept projections in the one-ball and twoball cases (e.g., focusing on speed and speed changes in oneball animations, versus on relative position and conservation of energy in the two-ball animations), a trend that has been observed in other physics reasoning experiments. ${ }^{20}$ What the physics students revealed during their interviews was, perhaps, a series of snapshots of developing knowledge systems. Nevertheless, both the physics students and psychology students did attempt to coordinate knowledge, with physics students displaying attempts to coordinate knowledge learned in physics class.

\section{CONCLUSION}

An analysis of the interview data of students' reasoning about the realism of animated motion resulted in the identification of suitable coordination system candidates (e.g., realism of motion, speed and speed changes, position). The performance specifications (alignment and span) for coordination classes, however, were not met by either group of students. Due to the complexity of the coordination systems studied, the observed lack of alignment and span had multiple sources, including incorrect readouts of various quanti- ties, the use of inappropriate readout strategies, and inappropriate causal net elements (i.e., expectations) and reasoning, all of which were highly sensitive to context. The inherent complexity of students' knolwedge systems makes it difficult to predict how learning will impact their coordination and use of knowledge, as evidenced by the way the physics knowledge of the physics students affected their approach to the two ball context. The coordination analysis presented here highlights the fact that learning in a domain like physics is a complex endeavor requiring considerable time, instructional resources, and appreciation of the complexity of applying ideas across diverse contexts.

These points should serve as a warning against the use of performance measures as the only means of assessing learning. Looking beyond performance, the physics students brought to bear more, and more sophisticated, causal net elements, attempted to justify them more thoroughly (despite errors in their attempts), and made more attempts to integrate their knowledge, than did the less-physics-knowledgeable psychology students. We view these mental habits as positive, even though the overall performance of the physics students was inconsistent at best. The significance of the physics students' poor overall performance on the two-ball task relative to the psychology students is mitigated by the realization that those performance differences can be attributed largely to an overextension of a major physics concept by the physics students (namely, that just because conservation of energy dictates that both balls should have the same speed on the final shelf does not mean that they will arrive at the end of the final shelf at the same time).

One of the challenges to using the coordination class construct is that it is difficult to draw a boundary around a particular coordination class. It is also likely that, among beginning science students, coordination systems related to technical concepts are unlikely to be fully functioning coordination classes. Even in the absence of coordination classes, however, we find that the coordination class construct provides a useful framework for analysis and explication of (even some surprising) student behavior. It offers a language and structure within which to gauge the fine-grained and complex progression of conceptual development and knowledge coordination along the expertise dimension. Although we know a considerable amount about the nature of expertise ${ }^{21}$ this knowledge largely comes from exploring the two end points of the dimension, namely, the performance of experts and novices in cognitive tasks. The model of finegrained coordination class analysis of students' thought processes should be further articulated and extended to more and more varied situations. As that happens, it will be overlaid with traditional research tools used in physics education research (e.g., assessments, think-aloud interviews), to help us design experiments and analyses that will yield valuable insights about progression toward expertise.

\section{ACKNOWLEDGMENTS}

This work was supported in part by NSF Grants No. DGE-9452801 and No. REC-0106771. 
*Author to whom correspondence should be addressed. Email address: mestre@uiuc.edu

${ }^{1}$ Reinders Duit, Bibliography: Students' Alternative Frameworks and Science Education (Leibniz Institute for Science Education, University of Kiel, Report 2004) (unpublished).

${ }^{2}$ John Clement, Students' preconceptions in introductory mechanics, Am. J. Phys. 50, 66 (1982).

${ }^{3}$ Stella Vosniadou and William F. Brewer, Mental models of the earth: A study of conceptual change in childhood, Cogn. Psychol. 24, 535 (1992).

${ }^{4}$ Christos Ioannides and Stella Vosniadou, The changing meanings of force, Cognit. Sci. Q. 2, 5 (2002).

${ }^{5}$ Lillian C. McDermott, Research on conceptual understanding in mechanics, Phys. Today 37 (7), 24 (1984), and references therein.

${ }^{6}$ Jose P. Mestre, in Teacher Enhancement for Elementary and Secondary Science and Mathematics: Status, Issues and Problems, edited by S. J. Fitzsimmons (National Science Foundation, Washington, DC, 1994), p. 3-1, and references therein.

${ }^{7}$ Andrea A. diSessa and Bruce L. Sherin, What changes in conceptual change, Int. J. Sci. Educ. 20, 1155 (1998).

${ }^{8} \mathrm{~S}$. Carey, in Conceptual Development: Piaget's Legacy, edited by Ellin K. Scholnick (Erlbaum, Mahwah, NJ, 1999), p. 293.

${ }^{9} \mathrm{G}$. Hatano and K. Inagaki, in The Handbook of Education and Human Development: New Models of Learning, Teaching, and Schooling, edited by David R. Olson (Blackwell, Cambridge, MA, 1996), p. 683.

${ }^{10}$ Michael McCloskey, in Mental Models, edited by Dedre Gentner (Erlbaum, Hillsdale, NJ, 1983), p. 299.

${ }^{11}$ Andrea A. diSessa, Toward an Epistemology of Physics, Cogn. Instruct. 10, 105 (1993).

${ }^{12}$ David Hammer, Andrew Elby, Rachel E. Scherr, and Edward F. Redish, in Transfer of Learning from a Modern Multidisci- plinary Perspective, edited by Jose P. Mestre (Information Age, Greenwich, CT, 2005), p. 89.

${ }^{13}$ Andrea A. diSessa and Joseph F. Wagner, in Transfer of Learning from a Modern Multidisciplinary Perspective (Ref. 12), p. 121.

${ }^{14}$ Jose P. Mestre, Thomas C. Thaden-Koch, Robert J. Dufresne, and William J. Gerace, in Research on Physics Education, Proceedings of the International School of Physics "Enrico Fermi," edited by Edward F. Redish (IOS Press, Amsterdam, 2004), p. 367.

${ }^{15}$ Michael C. Wittmann, The object coordination class applied to wave pulses: Analyzing student reasoning in wave physics, Int. J. Sci. Educ. 24, 97 (2002).

${ }^{16}$ We acknowledge that alternate models of student cognition might be productively used to analyze the experimental data, but will limit our inquiry to the use of coordination theory.

${ }^{17}$ Thomas C. Thaden-Koch, A Coordination Class Analysis of College Students' Judgments About Animated Motion, Ph.D. thesis, University of Nebraska-Lincoln, 2003.

${ }^{18}$ Students referred to each animation by number, corresponding to the virtual button they pressed to view it. The numbers varied from student to student, so we have annotated their comments with the animation's abbreviation (see Fig. 1) in square brackets.

${ }^{19}$ This excerpt comes from the "two-ball flat-tracks" section of the interview described in Robert J. Dufresne, Jose P. Mestre, Thomas C. Thaden-Koch, William J. Gerace, and William J. Leonard, in Transfer of Learning from a Modern Multidisciplinary Perspective, edited by Jose P. Mestre (Information Age, Greenwich, CT, 2005), p. 155.

${ }^{20}$ Andrea A. diSessa, Andrew Elby, and David Hammer, in Intentional Conceptual Change, edited by Gale M. Sinatra (Erlbaum, Mahwah, NJ, 2003), p. 237.

${ }^{21}$ John D. Bransford, Ann L. Brown, and Rodney R. Cocking, How People Learn: Brain, Mind, Experience, and School (National Academy Press, Washington, DC, 1999). 